\title{
Social Determinants of Health Mediate COVID-19 Disparities in South Florida
}

\author{
Ana Palacio, MD, $\mathrm{MPH}^{1,2}$ and Leonardo Tamariz, MD, $\mathrm{MPH}^{2,3}{ }_{\odot}$ \\ 'Department of Public Health Sciences, University of Miami, Miami, FL, USA; ${ }^{2}$ GRECC, Geriatric Research Education and Clinical Center, Veterans \\ Affairs Medical Center, Miami, FL, USA; ${ }^{3}$ Division of Cardiology, University of Miami, Miami, FL, USA.
}

BACKGROUND: There are several reports of health disparities related to COVID-19. Understanding social determinants of health $(\mathrm{SDoH})$ could help develop mitigation strategies to prevent further COVID-19 spread. Our aim is to evaluate self-reported and census-based $\mathrm{SDoH}$ as a mediator of health disparities in COVID-19.

METHODS: We conducted a cross-sectional ecological study and included all COVID-19 cases report by the COVID-19 Florida dashboard as the dependent variable. The independent variables were census-based median household income, population and household size, and self-reported SDoH using a validated survey. We calculated the incidence rate ratio (IRR) of COVID-19 by zip code using Poisson regression and structured equation modelling to evaluate the mediation effect of income and $\mathrm{SDoH}$ on COVID- 19 cases.

RESULTS: We included 97,594 COVID-19 positive cases across 79 Miami-Dade ZIP codes with a median age of 43 years; females represented $50.7 \%$ of the cases. The highest IRR (4.44) were for ZIP code 33125 (income $\$ 21,106,6 \%$ Black, 93\% Hispanic), while the lowest IRR (0.86) was for ZIP code 33146 (median household incomes $\$ 96,609,3 \%$ Black and 53\% Hispanic). In structured equation models, the indirect coefficient of income in the relationship between race/ethnicity and COVID-19 were only significant for Blacks and not Hispanics.

CONCLUSIONS: This ecological analysis using ZIP code and aggregate individual-level $\mathrm{SDoH}$ shows that in MiamiDade county, COVID infection is associated with economic disadvantage in a particular geographical area and not with racial/ethnic distribution.

J Gen Intern Med 36(2):472-7

DOI: $10.1007 /$ s11606-020-06341-9

(C) Society of General Internal Medicine 2020

\section{INTRODUCTION}

In New York and Chicago, the age-adjusted COVID-19 mortality is greater for Blacks and Hispanics compared with NonHispanic Whites. ${ }^{1}$ The same trends are emerging in several other states as the pandemic spreads through the USA. ${ }^{2}$ A recent study from a large health system in California found

Social Determinants of Health Mediate COVID-19

Supplementary Information The online version contains supplementary material available at https://doi.org/10.1007/s11606-020-06341-9.

Received June 2, 2020

Accepted October 31, 2020

Published online November 18, 2020 that African American patients were more than twice as likely to be hospitalized for COVID-19 when compared to NonHispanic White patients. Government officials and scientific journals call for large-scale contextualization of the COVID19 data to understand these disparities and avoid misunderstandings that undermine efforts to reduce inequities. ${ }^{3}$ Even though a growing number of voices are recognizing the role that social determinants of health $(\mathrm{SDoH})$ may be playing in the COVID-19 health disparities, there is a lack of consistent data to evaluate and understand these relationships. ${ }^{1}$ Ultimately, this limitation may not only impede the development of effective strategies to mitigate the pandemic ${ }^{2}$ but may also increase the risk of reigniting biological and stereotypical explanations of disparity that have limited scientific basis, distract from the real discriminatory culprits of inequity, and lead to stigmatization of communities. ${ }^{3}$ In order to preempt these dangers, Chowkwanyun et al. recommend to collect or, if needed, impute socio-economic, racial, and other $\mathrm{SDOH}$ data on COVID-19 cases. ${ }^{3}$ This would allow us to explore the interplay between race/ethnicity and SDOH that lead not only to COVID-19 disparities but also to the comorbid conditions reported to affect COVID-19 morbidity and mortality. ${ }^{2}$ Describing these relationships would advance the understanding on how social factors get "embodied" and alter biological processes resulting in disease. ${ }^{4}$

We aim to explore the social context of the pandemic through an ecological study correlating $\mathrm{SDoH}$ and the distribution of COVID-19 cases in Miami-Dade county, in South Florida. Miami-Dade offers an excellent natural experiment opportunity to tackle this aim since it is a diverse minoritymajority county that is currently one of the COVID-19 epicenters.

\section{METHODS}

\section{Study Design and Setting}

We conducted a cross-sectional ecological study in MiamiDade county. Miami-Dade is located in South Florida and has a population of 2,834,352 inhabitants of whom $17 \%$ are Black, $71 \%$ are Hispanics, and $12 \%$ are Non-Hispanic White. The county has a median household income of $\$ 49,239$ IQR $(40,039-61,897)$. Stratified by race, the median household income is $\$ 56,791$ for White and $\$ 40,162$ for Black residents 
and by ethnicity is $\$ 50,730$ for Hispanics and $\$ 60,268$ for Non-Hispanics.

\section{Definition of COVID-19}

We considered a positive case of COVID-19 if it was reported in Miami-Dade County by July 24, 2020. We used the Florida's COVID-19 data and surveillance dashboard to ascertain COVID-19 cases (https://experience.arcgis.com/experience/96dd742462124fa0b38ddedb9b25e429). The dashboard reports the cumulative number of positive SARS $\mathrm{CoV}-2$ tests by county and by Zone Improvement Plan (ZIP) code.

\section{Definition of Race/Ethnicity}

Although the Florida COVID-19 dashboard reports race and ethnicity, $50 \%$ of all cases have missing race/ethnicity data. For this reason, we imputed race/ethnicity from each ZIP code using census data. ${ }^{5}$ For all analyses including the calculation of incidence rate ratios, we used the proportion of each race and ethnicity in each ZIP code as a continuous variable. To calculate and report an estimate of incidence by race and ethnicity, we assigned these attributes based on the ZIP code distribution. We assigned Hispanic ethnicity to cases residing in ZIP codes with $>50 \%$ Hispanics and $<30 \%$ Black. We assigned Black race to cases residing in all ZIP codes with $>$ $30 \%$ Black residents and cases in all other distributions were considered non-Hispanic White. ${ }^{6}$ We used 2010 census data to describe median household income, race, and ethnicity distributions by ZIP code.

\section{Social Determinants of Health}

We included two sources of SDoH data. The first source was ZIP code-level data from the 2010 Census population data using the Miami-Dade projections (http://www. miamidadematters.org/demographicdata). We used total population, race and ethnicity, median household income, and average household size by ZIP code to match how the COVID cases are reported in the state of Florida. The second source was individual-level self-reported SDoH from a survey of 11,113 primary care UHealth patients who responded to a validated questionnaire based on the recommendations of the National Academy of Medicine. ${ }^{7}$ The survey was collected between September 2016 and April of 2019. The individuallevel data included race and ethnicity, education, financial strain, stress, social isolation scale,${ }^{8}$ health literacy, and delays in receiving health care (appendix Table 1). ${ }^{9-11}$ In order to define if a specific social risk was present or absent at the individual level, we dichotomized the answer to each question based on existing literature on each of the validated questions (appendix Table 2). This assignment was necessary to aggregate the prevalence of each SDOH at the ZIP code level. All variables of the survey were used individually except for variables related to social isolation which form a scale. Survey responders received 1 point for risks defined as present as follows: living alone, having high school education or less, stating that it was somewhat hard or very hard to pay for basic needs such as food or medications, feeling not confident, little or somewhat confident filling out forms, feeling quite a bit or very much stress, delaying care at least once in the past 12 months, and for the social isolation scale having a score of 3 or 4.

\section{Statistical Analysis}

To evaluate the incidence of COVID-19 per ZIP code, we used Poisson regression to calculate the incidence rate ratios (IRR) adjusted for total population and household size by ZIP code using as a reference ZIP code 33158 (\$145,541 income, 2.7\% Black, $47 \%$ Hispanic, and 50\% non-Hispanic White). We did not use the ZIP code with the highest income because it corresponds to a very restricted geographical area with a very small population (33109).

For both ZIP code and aggregated individual-level data, we first completed univariate linear regressions to fit each $\mathrm{SDOH}$ with the IRR for COVID-19. To evaluate the relationships between COVID-19 and SDoH, we used multivariate linear regression. The dependent variable for this analysis was the number of COVID-19 cases and the independent variable were all the SDoH factors collected from the census (ZIP code level) and from the SDoH survey (aggregated individual-level).

We divided the median household income into quartiles and the self-reported individual-level financial strain into tertiles and calculated the IRR using Poisson regression for each subgroup.

We used structured equation models to evaluate the mediation effect of significant census or self-reported $\mathrm{SDoH}$ on the relationship between race/ethnicity and COVID-19 incidence. We stratified the models for Blacks and Hispanics and included continuous variables. We computed indirect and total effects and reported the coefficient and $p$ values of the indirect effect as the mediation effect.

\section{RESULTS}

\section{Incidence of COVID-19 Infection in Miami- Dade County by ZIP Code}

For this analysis, we included 79 of the 80 Miami-Dade ZIP codes because one ZIP code (33199) did not report any

Table 1 Incident Rate Ratios by Race Stratified by Median Household Income

\begin{tabular}{llll}
\hline \hline $\begin{array}{l}\text { Race/ } \\
\text { ethnicity }\end{array}$ & $\begin{array}{l}\text { COVID-19 } \\
\text { incidence per } \\
\mathbf{1 0 0 0}\end{array}$ & $\begin{array}{l}\text { COVID-19 } \\
\text { incidence per } \\
\mathbf{1 0 0 0} \text { if income } \\
\mathbf{\$ 5 0 , 0 0 0}\end{array}$ & $\begin{array}{l}\text { COVID-19 } \\
\text { incidence per } \\
\mathbf{1 0 0 0} \text { if income } \\
\mathbf{\$ 5 0 , 0 0 0}\end{array}$ \\
\hline White & $37.1 \pm 10.3$ & $43.4 \pm 31.6$ & $33.5 \pm 37.6$ \\
Black & $43.2 \pm 43.2$ & $47.0 \pm 69.3$ & $33.6 \pm 22.1$ \\
Hispanic & $38.2 \pm 26.3$ & $43.3 \pm 31.2$ & $35.2 \pm 40.7$ \\
\hline
\end{tabular}


census-based SDoH or COVID-19 cases. As of July 24, 2020, there were 97,594 unique positive tests for SARS CoV-2 representing 34.4 cases per 1000 Miami-Dade residents. Stratified by race and ethnicity, the rate per 1000 residents was 38.9 \pm 36.6 for Hispanics, $47.6 \pm 50.7$ for Blacks, and $31.6 \pm 11.5$ for non-Hispanic Whites (Table 1).

\section{Baseline Characteristics of Positive Cases}

Our study included 97,594 COVID-19 positive cases across 79 Miami-Dade ZIP codes with a median age of 43 years; females represented $50.7 \%$ of the cases $(n=49,538)$. As of July 24, there were 9382 (9\%) cases among Black residents and 33,181 (34\%) cases among White residents. By ethnic group there were 36,547 cases among Hispanics and 11,711 cases (12\%) among non-Hispanics. Fifty percent of all cases had missing race/ethnicity information (Table 3 of the appendix).

\section{Relationship Between COVID-19 Infection and SDOH}

The IRR and the census-based demographic data by each ZIP code are detailed in Table 3 of the appendix. The highest IRR (4.44) were for ZIP code 33125 (income \$21,106, 6\% Black, 93\% Hispanic), and the second highest IRR (4.25) was for ZIP code 33128 (\$22,644, 12\% Black and 88\% Hispanic). In contrast, the 2 ZIP codes with the lowest IRR had significantly higher median household income. The lowest IRR $(0.86)$ was for ZIP code 33146 (median household incomes \$96,609, 3\% Black and 53\% Hispanic). The second lowest IRR (0.99) was for ZIP code 33154 (median household income \$76,342, Black 3\%, and Hispanic 51\%).

To illustrate the relationships between SDOH and COVID19 rates, we depict the SDoH characteristics of three MiamiDade ZIP codes with high, intermediate, and low COVID-19 IRR (Fig. 1). ZIP codes with high and intermediate IRR tended to have lower household income, higher financial strain, and more social isolation. The neighborhood with one of the lowest rates of COVID-19 had the largest representation of non-Hispanic White residents (38\%), while the one with highest COVID-19 rate had a high representation of Black residents. Hispanics were significantly represented in all ZIP codes.

In multivariate models, median household income and selfreported individual-level financial strain and stress were the only significant predictors of incident COVID-19 (Table 2). Population density was significant in both univariate and multivariate analysis but not in the multivariate models.

\section{Relationships Between COVID and Socio- economic Status (SES)}

To further explore the relationship between COVID-19 and SES, we compared IRR by quartiles of median household income (Table 3). In models adjusted for population and household size, the IRR increased as the median household income by ZIP code decreased. The lowest quartile of income had an IRR of 1.89 (1.85-1.93), while the IRR for second quartile of income was $1.16(1.13-1.19)$.

We also compared the IRR across ZIP codes with different self-reported burden of financial strain. Table 3 shows the IRR of having COVID-19 by tertiles in the proportion of responders self-reporting significant financial distress. ZIP codes with the highest number of responders with financial strain (highest tertile) had an IRR of 1.51 (1.49-1.54), while the IRR for the responders in the intermediate tertile was 1.17 (1.15-1.19).

To explore the effect of SES on the disparate rates of COVID-19 infection, we first stratified the incidence rate ratios by median household income above or below $\$ 50,000$ (Table 3). The racial differences in IRR were mitigated in the stratified analysis. The IRR for Black Miami-Dade residents decreased from $47.6 \pm 50.7$ to $33.6 \pm 22$ when the median household income in the ZIP code was $>\$ 50,000$. Similarly, the IRR for Hispanics and non-Hispanic Whites increased for ZIP codes with an average median household income of less than $\$ 50,000$.

We used structured equation models to evaluate if socio-economic status and stress mediated the racial disparities. These models revealed that the indirect coefficient of income between the relationship between Blacks and COVID-19 was $6.63(p<0.01)$ while for financial distress was $3.82(p=0.04)$. The indirect coefficient of income between the relationship between Hispanics and COVID-19 was $2.29(p=0.24)$ while for financial distress was $2.9(p=0.09)$. Figure 1 of the appendix shows the SEM coefficients. The indirect coefficients for stress between Black race and Hispanic ethnicity and IRR for COVID-19 were non-significant.

\section{DISCUSSION}

This ecological analysis using ZIP code and aggregate individual-level SDoH shows that in Miami-Dade county COVID-19 infection is associated with the economic disadvantage and stress reported in a particular geographical area and not with its racial/ethnic distribution. Other researchers have reported the significant role of place of residence in COVID-19 outcomes. ${ }^{12,13}$ Our data contributes to the COVID-19 disparity literature by highlighting that socioeconomic measures such as the zip code-level median household income and self-reported financial strain mediate the relationship between Black race and COVID-19 infection. Another important contribution is the finding that selfreported stress aggregated to the ZIP code level is directly associated was COVID-19 infection. As previously reported for Manhattan, ${ }^{12}$ population density was not a significant correlate of COVID-19 infection in Miami-Dade County. 


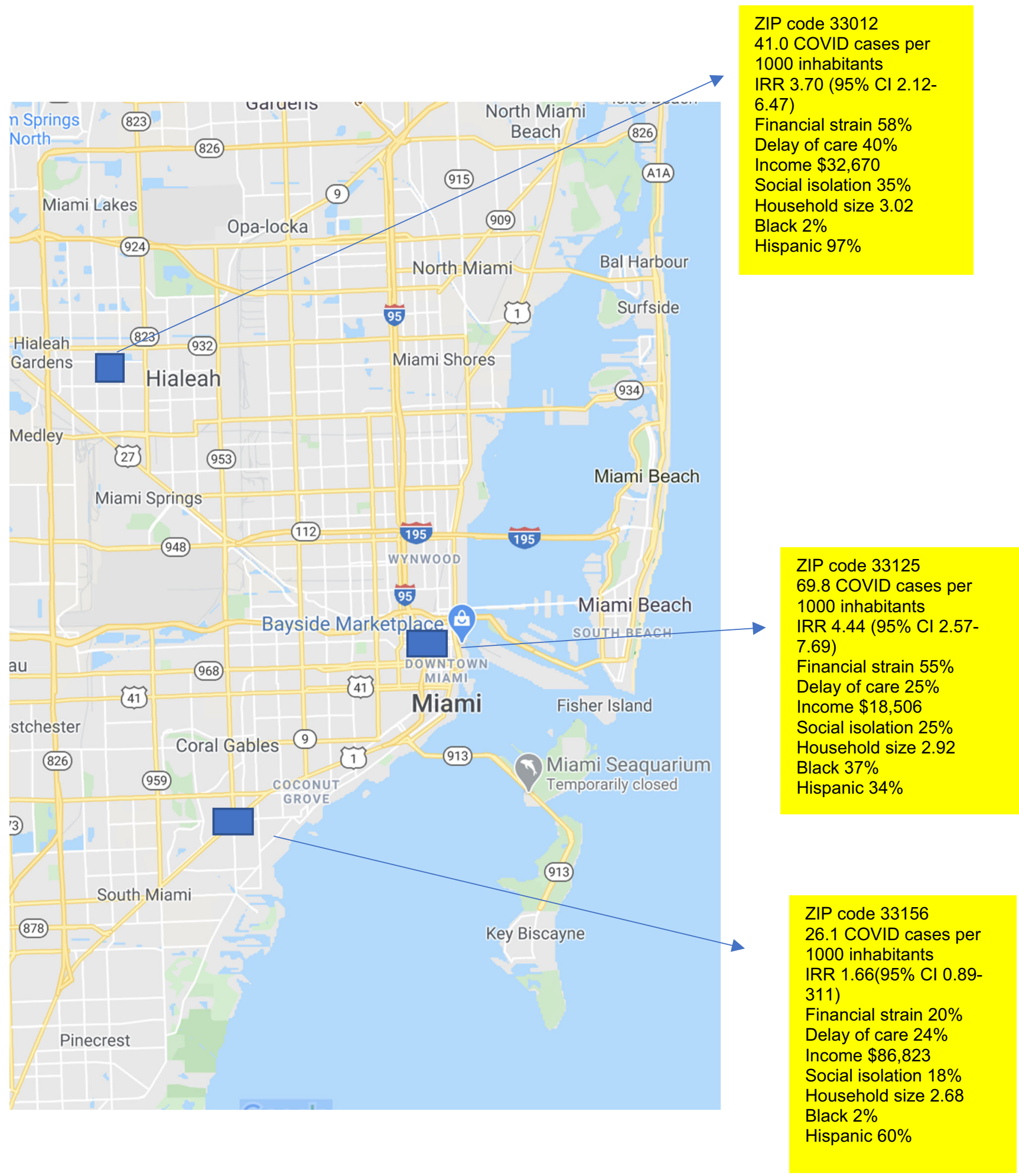

Figure 1 Relationship between COVID-19 cases and social determinants of health in three representative ZIP codes in Miami-Dade county.

The mechanisms by which median household income, stress, and financial stress can impact COVID-19 infection rates have already been highlighted in the literature.

Potential explanations for the expanding evidence of COVID-19 disparities generally include the higher prevalence of comorbidities among racial/ethnic minority populations and the socio-economic factors that may affect minorities' ability to social distance. ${ }^{1}$

The higher prevalence of cardiovascular disease, diabetes, and obesity among racial/ethnic minorities has been extensively associated with the same social determinants of health ${ }^{14,15}$ we collected for this study. Moreover, there is 
Table 2 Multivariate Model of IRR for COVID-19

\begin{tabular}{lll}
\hline \hline Variable & $\begin{array}{l}\text { Model A with median } \\
\text { household income beta } \\
\text { coefficient and } \boldsymbol{p} \text { value }\end{array}$ & $\begin{array}{l}\text { Model B with financial } \\
\text { strain beta coefficient } \\
\text { and } \boldsymbol{p} \text { value }\end{array}$ \\
\hline $\begin{array}{l}\text { Income } \\
\text { Financial }\end{array}$ & $-0.0015(<0.01)$ & $\mathrm{NA}$ \\
$\begin{array}{l}\text { strain } \\
\text { Social }\end{array}$ & $-0.06(0.66)$ & $0.28(<0.01)$ \\
isolation & $0.007(0.93)$ & $-0.08(0.54)$ \\
$\begin{array}{l}\text { Black } \\
\text { Hispanic }\end{array}$ & $0.05(0.25)$ & $0.019(0.82)$ \\
$\begin{array}{l}\text { Delay in } \\
\text { care }\end{array}$ & $0.16(0.24)$ & $0.01(0.87)$ \\
Stress & $0.22(0.04)$ & $-0.02(0.87)$ \\
$\begin{array}{l}\text { Household } \\
\text { size }\end{array}$ & $2.5(0.29)$ & $0.21(0.04)$ \\
Population & $0.31(<0.01)$ & $1.5(0.50)$ \\
\hline
\end{tabular}

growing evidence that these factors may become stressful stimuli that produce a cascade of immunological, neuroendocrine, and cardiovascular responses that can disrupt biologic pathways ${ }^{16}$ such as the hypothalamic pituitary adrenalcortisol axis. ${ }^{4}$ These disruptions may lead to chronic disease and inflammation and make people more susceptible to infections. Our finding that stress was associated with COVID-19 infection supports this possible mechanism. We need studies to confirm the mechanisms through which $\mathrm{SDoH}$ produce higher comorbidity but the data to date support the need to evaluate social and structural determinants of health, racism, and discrimination as culprits of disparities in COVID-19 outcomes, from infection to mortality.

Our findings also support the social distancing hypothesis. The groups at most risk of COVID-19 infection had very low median household income according to ZIP code-level data and also had a higher proportion of people reporting financial difficulties paying for basic needs such as food or medications. Groups with these characteristics are potentially less likely to have the privilege of staying at home for long periods of time and more likely to be exposed for work related demands. ${ }^{17}$

In contrast to our findings among Black residents, we did not find an excess rate of COVID-19 infection among Hispanics when compared to non-Hispanic Whites and socioeconomic variables did not mediate the relationship between ethnicity and incidence of a positive SARS CoV-2 test. This may be related to several factors such as the Hispanic paradox ${ }^{18-21}$ or that Hispanics are the largest ethnic group in Miami-Dade county with a higher socio-economic position

Table 3 Incidence Rate Ratios by Quartile of Income and by Tertile of Self-Reported Financial Strain

\begin{tabular}{lll}
\hline \hline Quartile & Income \$ & IRR (95\% CI) \\
\hline 1 (Reference) & $62,170-192,500$ & 1 \\
2 & $50,194-61,897$ & $1.16(1.13-1.19)$ \\
3 & $41,541-49,239$ & $1.46(1.43-1.50)$ \\
4 & $14,999-40,039$ & $1.89(1.85-1.93)$ \\
Tertile & Financial strain \% & IRR (95\% CI) \\
1 (Reference) & $0-32$ & 1 \\
2 & $34-52$ & $1.17(1.15-1.19)$ \\
3 & $53-100$ & $1.51(1.49-1.54)$ \\
\hline
\end{tabular}

when compared to Black residents. Nevertheless, reports from other states have also described that COVID-19 disparities are occurring disproportionately within the Black community. ${ }^{13}$ We need more data from other geographical areas to evaluate the impact of COVID-19 among Hispanics and to evaluate potential risk or resilience factors.

Our study has several limitations. First, our ecological design has a narrow geographical focus. Second, COVID-19 numbers are reported by ZIP codes and not by block groups or census tracks; this could lead to misclassification. Third, Hispanics frequently identify as of White race in the census-based question which may lead to misclassification.

Nevertheless, until larger scale individual-level data is available, the findings originating from multiple cities support the calls to address SDoH. ${ }^{22}$ The CDC compiled a list of actions that community organizations, health systems, and individuals could undertake to mitigate COVID disparities. In the long term, only increasing awareness and empathy on the root causes of disparities among the general public will allow the creation of truly equitable health policies. ${ }^{22}$

White/Black comparison $p=0.03$

White/Hispanic comparison $p=0.35$

Adjusted for population and household size

Corresponding Author: Ana Palacio, MD, MPH; GRECC, Geriatric Research Education and Clinical Center, Veterans Affairs Medical Center, Miami, FL, USA (e-mail: apalacio2@miami.edu).

Funding The research reported in this publication was supported by COVID-19 Rapid response grant UM 2020-2231 and NIMHD and NHGRI of the National Institutes of Health under award number U54MD010722.

\section{Compliance with Ethical Standards:}

Conflict of Interest: The authors declare that they do not have a conflict of interest.

\section{REFERENCES}

1. Webb Hooper M, Napoles AM, Perez-Stable EJ. COVID-19 and racial/ ethnic disparities. JAMA. 2020.

2. Owen WF, Jr, Carmona R, Pomeroy C. Failing another national stress test on health disparities. JAMA. 2020.

3. Chowkwanyun M, Reed AL, Jr. Racial health disparities and COVID-19 - caution and context. N Engl J Med. 2020.

4. Palmer RC, Ismond D, Rodriquez EJ, Kaufman JS. Social determinants of health: future directions for health disparities research. Am J Public Health. 2019; 109(S1):S70-1.

5. Tamariz L, Medina H, Suarez M, Seo D, Palacio A. Linking census data with electronic medical records for clinical research: a systematic review. J Econ Soc Meas. 2018;43(1-2):105-18.

6. Henry KA, Boscoe FP. Estimating the accuracy of geographical imputation. Int $J$ Health Geogr 2008;7:3,072X-7-3.

7. Adler NE, Glymour MM, Fielding J. Addressing social determinants of health and health inequalities. JAMA. 2016;316(16):1641-2.

8. Pantell M, Rehkopf D, Jutte D, Syme SL, Balmes J, Adler N. Social isolation: a predictor of mortality comparable to traditional clinical risk factors. Am J Public Health. 2013;103(11):2056-62. 
9. Palacio AM, Seo D, Suarez M, Tamariz L. Integrating social determinants of health into the electronic health records of a large health system: a qualitative perspective. 2018 .

10. Palacio A, Seo D, Medina H, Singh V, Suarez M, Tamariz L. Provider perspectives on the collection of social determinants of health. Popul Health Manag. 2018;21(6):501-8.

11. Palacio A, Suarez M, Tamariz L, Seo D. A road map to integrate social determinants of health into electronic health records. Popul Health Manag. 2017;20(6):424-6.

12. Wadhera RK, Wadhera $\mathbf{P}$, Gaba $\mathbf{P}$, et al. Variation in COVID-19 hospitalizations and deaths across New York City boroughs. JAMA. 2020.

13. Azar KMJ, Shen Z, Romanelli RJ, et al. Disparities in outcomes among COVID-19 patients in a large health care system in California. Health Aff (Millwood). 2020;39(7): 1253-62

14. Knighton AJ, Stephenson B, Savitz LA. Measuring the effect of social determinants on patient outcomes: a systematic literature review. J Health Care Poor Underserved. 2018;29(1):81-106.

15. Havranek EP, Mujahid MS, Barr DA, et al. Social determinants of risk and outcomes for cardiovascular disease: a scientific statement from the american heart association. Circulation. 2015;132(9):873-98.

16. Berkman LF, Macintyre S. The measurement of social class in health studies: old measures and new formulations. IARC Sci Publ 1997;138, (138):51-64.

17. Yancy CW. COVID-19 and African Americans. JAMA. 2020.

18. Damas OM, Estes D, Avalos D, et al. Hispanics coming to the US adopt US cultural behaviors and eat less healthy: implications for development of inflammatory bowel disease. Dig Dis Sci. 2018.

19. Damas OM, Gomez L, Quintero MA, et al. Genetic characterization and influence on inflammatory bowel disease expression in a diverse Hispanic South Florida cohort. Clin Transl Gastroenterol. 2017;8(4):e87.

20. Luchsinger JA, Cabral R, Eimicke JP, Manly JJ, Teresi J. Glycemia, diabetes status, and cognition in Hispanic adults aged 55-64 years. Psychosom Med. 2015;77(6):653-63.

21. Balfour PC, Jr, Ruiz JM, Talavera GA, Allison MA, Rodriguez CJ. Cardiovascular disease in Hispanics/Latinos in the United States. J Lat Psychol. 2016;4(2):98-113.

22. Williams DR, Cooper LA. COVID-19 and health equity-a new kind of "herd immunity"|. JAMA. 2020.

Publisher's Note: Springer Nature remains neutral with regard to jurisdictional claims in published maps and institutional affiliations. 